\title{
OBSERVAR A LOS NIÑOS Y NIÑAS ES APRENDER DE ELLOS. UNA EVALUACIÓN EN EDUCACIÓN INFANTIL CENTRADA EN LOS NIÑOS Y NIÑAS.
}

\author{
TO OBSERVE CHILDREN IS TO LEARN FROM THEM. \\ CHILD-CENTERED ASSESSMENT IN EARLY CHILDHOOD EDUCATION.
}

Mónica Manhey Moreno ${ }^{1}$

Doctora en Educación

Universidad de Granada.

mmanhey@u.uchile.cl

Resumen: Muchas veces al recordar la enseñanza que tuvimos, afloran buenos y malos momentos, y entre ellos las experiencias evaluativas, algunas gratificantes y otras decepcionantes y hasta humillantes. Revisando la evaluación en el sistema escolar ha sido utilizada para privilegiar una manera de ser y hasta de definir a un estudiante modelo, fabricando jerarquías (Perrenoud, 2010) ello ha producido efectos en la vida de las personas y la educación inicial no ha estado exento de ello. En este escrito nos referiremos a la evaluación que se realiza en el aula o algún sistema de tipo no convencional, pero en un nivel micro, es decir, no de la gestión, ni del sistema, sino para el aprendizaje de los niños y niñas menores de seis años. Invitamos por tanto al lector a remirar las prácticas pedagógicas y otorgarle mayor relevancia a detenerse, observar y aprender de los niños y niñas y sus formas de relacionarse y aprender, sin mostrar un modelo ideal. Abordaremos la importancia de una observación abierta, donde el educador además de plantearse objetivos de aprendizaje a priori, pueden emerger otros, posterior a la observación de los niños y niñas, todo ello si tiene una apertura y pueda mirar sin "lentes" que le nublen la mirada y la cierren a lo preestablecido.

Palabras claves: evaluación, observar, infancia

Abstract: Many times, when remembering the teaching we had, good and bad moments come to the surface, and among them, the evaluative experiences, some gratifying and others disappointing and even humiliating. Reviewing the evaluation in the school system, it has been used to privilege a way of being and even to define a model student, creating hierarchies (Perrenoud, 2010). This has produced effects on people's lives and initial education has not been exempt from this. In this paper, we will refer to the evaluation that happens in the classroom or some non-conventional type of system, but at a micro level, that is, not of the management, nor the system, but for the learning of children under six

${ }^{1}$ Académica Departamento de Educación, Facultad de Educación, Universidad de Chile 
years of age. Therefore, we invite the reader to rethink pedagogical practices and give greater relevance to stopping, observing, and learning from children and their ways of relating and learning, without showing an ideal model. We will address the importance of an open observation, where the educator, besides setting learning objectives beforehand, can allow others to emerge after observing the children, if he/she is open and can look without "lenses" that cloud his/her gaze and close it to the pre-established.

Key words: evaluation, observation, childhood

\section{INTRODUCCIÓN}

Muchas veces al recordar la enseñanza que tuvimos, afloran buenos y malos momentos, y entre ellos las experiencias evaluativas, algunas gratificantes y otras decepcionantes y hasta humillantes. Revisando la evaluación en el sistema escolar ha sido utilizada para privilegiar una manera de ser y hasta de definir a un estudiante modelo, fabricando jerarquías (Perrenoud, 2010) ello ha producido efectos en la vida de las personas y la educación inicial no ha estado exento de ello. En este escrito nos referiremos a la evaluación que se realiza en el aula o algún sistema de tipo no convencional, pero en un nivel micro, es decir, no de la gestión, ni del sistema, sino para el aprendizaje de los niños y niñas menores de seis años. Invitamos por tanto al lector a remirar las prácticas pedagógicas y otorgarle mayor relevancia a detenerse, observar y aprender de los niños y niñas y sus formas de relacionarse y aprender, sin mostrar un modelo ideal. Abordaremos la importancia de una observación abierta, donde el educador además de plantearse objetivos de aprendizaje a priori, pueden emerger otros, posterior a la observación de los niños y niñas, todo ello si tiene una apertura y pueda mirar sin "lentes" que le nublen la mirada y la cierren a lo preestablecido.

\section{DESARROLLO}

La evaluación para el aprendizaje, más que de los aprendizajes, implica un proceso en que es de relevancia detenerse, observar y resguardar la singularidad de los niños y niñas, alejándose de instrumentos preconcebidos que ven a los sujetos como un objeto. Las prácticas pedagógicas también incluyen la evaluación que realizan los educadores, la que se constituye muchas veces en un problema especialmente para éstos. Las preguntas comunes son: ¿Qué evaluar? ¿Cómo? ¿Cada cuánto? ¿Con qué instrumento? A lo que además debieran preguntarse ¿Para qué evaluar? Como se señalaba en la introducción, generalmente, se ha utilizado la evaluación para ver el logro o no logro de objetivos, considerando a unos mejores que otros, sin tomar en cuenta la diversidad humana o la consideran de acuerdo con una jerarquía en la que unas conductas, formas de ser, pensar, sentir, pueden o son mejores que otras. (Manhey, 2014). Se hace por tanto necesario revisar las prácticas pedagógicas al respecto para evitar impactos negativos en los niños y niñas y, en estas edades, sobre todo a las familias. Éstos adultos familiares también han vivido bajo el sistema de jerarquías de 
excelencia, tienden a angustiarse si algo señala que su niño o niña no ha logrado algo, o si saben que hay un marco ideal al que no han llegado, o comparan los resultados con otros niños y niñas del grupo, que por cierto muchas veces son homogéneos en edades en estos niveles (entre 2 a 3 años, 3 a 4, 4 a 5 años...) y educadores escogen o utilizan o les demandan las autoridades institucionales utilizar instrumentos con conductas predeterminadas y por edades, lo que es altamente riesgoso el ver a los niños y niñas como iguales, ante ello nos preguntamos ¿todos los niños y niñas deberán de "lograr" lo mismo a cierta edad? Ello es una buena pregunta y ha generado discusiones desde antaño. Hoy que sabemos de la importancia del contexto sociocultural, de la importancia de las oportunidades de aprendizaje y de las diferencias individuales, es complejo "medir" con un mismo instrumento a todos los niños y niñas y más aún plantear indicadores preestablecidos. Al decir "medir" apunta a un paradigma positivista o también llamado paradigma cuantitativo, considerado el modelo dominante en las ciencias sociales hasta tiempos recientes y también presentes, donde los hechos o causas de los fenómenos son independientes de los estados subjetivos de los individuos.

Por lo expuesto, se hace necesario en la etapa de los niños y niñas menores de seis años donde no se debiera poner una calificación, revisar el contexto antes de emitir un juicio. Pese a ello, muchas veces se traducen los registros de observación de tipo cualitativo en criterios cuantitativos, tal vez no con una "nota" o calificación, (de 1 a 7 u otra norma como es el caso de otros países en que la nota máxima es 10 o 20), sin embargo, se traduce en conceptos que clasifican en "buenos", "regulares" o "deficientes" a quienes se observan. También existen prácticas en que se asignan las categorías de "Logrado, Por Lograr o No Logrado" como si el resultado esperado fuera uno sólo, todo ello nos habla de una concepción de enseñanza, de aprendizaje, y un concepto de persona hacia el que aprende, lo que comúnmente se relaciona con el estudiante, niños y/o niñas y no al educador, que aprende o debería aprender constantemente de quien observa.

La invitación es a recobrar la observación como se señaló en un inicio, sin prejuicios, y no a jerarquizar lo recogido, clasificando a los niños y niñas. Si hacemos una analogía, no hemos visto a ningún médico clasificando a los pacientes entre menos a más enfermos, o al que logra más o menos recuperación y menos aún en aplicarles un tratamiento o medicamento de forma colectiva, se esfuerza el facultativo por un tratamiento individual, según sus necesidades. (Perrenoud, 2010). De esta manera la evaluación educacional y especialmente en primera infancia debiera tener las mismas características, sin clasificar, 0 dar orientaciones, objetivos o estrategias iguales para todos y todas.

Las ciencias sociales se basan en la observación de los hechos, lo que le permite recabar datos y generar conocimiento, en educación donde el educador es un investigador de la realidad, la observación se transforma en la técnica básica utilizada para poder recabar información relevante, a fin de investigar las diferentes realidades que se dan en el ámbito educativo, siendo "un proceso de búsqueda donde no basta mirar, es preciso escudriñar". (Santos, 1998, p.57) No cabe duda de que ésta es la técnica más utilizada en educación parvularia para recoger información de los aprendizajes de los niños y niñas, sin embargo, muchas veces se observa con prejuicio, es decir con focos dados o indicadores en que apuntan qué observar, limitando este acto. Por tanto, es necesario observar sin prejuicios y 
aprendiendo a mirar en forma cuidadosa, así será una verdadera y útil fuente para conocer a los niños y niñas, sus gustos, sus necesidades de aprendizaje, intereses, las interacciones que se dan en el aula, así como los ambientes de aprendizaje por señalar ejemplos de tan amplia fuente de conocimientos.

A lo largo de los años la observación ha cobrado importancia siendo parte de la evaluación, fuente para recolectar información y para aprender de quienes se observan, requiriendo un examen cuidadoso, para posteriormente tomar decisiones acertadas. Por consiguiente, la evaluación es un "proceso que lleva a emitir un juicio respecto de uno o más atributos de algo o alguien, fundamentado en información obtenida, procesada y analizada correctamente y contrastada con un referente" (Himmel, 1999) el que no sólo debe ser teórico, sino sobre todo tomando como antecedente la historia de vida y el proceso vivido por los niños y niñas, en este caso. En tal sentido la observación está al servicio de la evaluación, y ésta del aprendizaje. Es un acto de conocimiento, pero no un conocimiento abstracto, se trata de una emoción del conocimiento que puede contener toda nuestra subjetividad, expectativas, aquello que esperamos que pase, nuestras hipótesis y nuestras teorías de referencia, en las cuales nosotros también estamos reflejados, (Davoli, 2011) lo que implica ciertos riesgos que deben considerarse. ya que depende de nuestro punto de vista., de nuestras preguntas previas y de nuestros imaginarios. Al respecto se puede afirmar por tanto, que la evaluación siempre va a partir de una indagación de algo que interesa, y para ello es fundamental la observación, sin embargo, no se puede quedar en esta etapa del proceso, ello es sólo el punto de partida, se requiere de un acto sistemático en el cual nos detengamos, se analice lo observado y cuestionemos, produciendo conocimiento, compartiéndolo con la comunidad educativa, especialmente, otros profesionales y técnicos que participan en el proyecto educativo, y con la familia, de tal manera de tomar decisiones que retroalimenten el proceso educativo.

En la década de los '80 se decía que la evaluación se refería a “un proceso que requiere atención voluntaria e inteligente, orientada por un objetivo terminal, u organizador y dirigido hacia un objeto para tener información" (De Kelete,1984, p.24). En este caso se limitaba a observar algo que se tenía previsto. Ello se relaciona con registros de observación en que a priori se establece qué mirar, por ejemplo, las Listas de Cotejo, Escalas de evaluación u otros instrumentos con conductas preestablecidas. Ejemplos indicadores tales como: responde a preguntas, salta en un pie, se sienta sin apoyo... Este concepto ha ido evolucionando en la forma antes mencionada, ampliando procedimientos a una evaluación "mosaical", es decir, utilizando diferentes tipos de estrategias para recoger información y de una manera más abierta, sin sesgos.

Al respecto se hace énfasis en que no cualquiera puede ser un buen observador, se requiere ciertas condiciones. Para observar sin "lentes", sin prejuicios, Loris Malaguzzi (1994) decía que los educadores aprenden por medio de la observación para después desarrollar sus propios proyectos de creación, para lo cual se requiere documentar la experiencia de los niños y niñas. De esta manera, en la documentación tienen más sentido los procesos que los resultados finales. Observación, documentación e interpretación constituyen tres partes inseparables de un mismo proceso, los documentos que se producen permiten día a día reflexionar, aprender, enriquecernos y tomar decisiones pertinentes. Es 
relevante aclarar que documentar incluye escribir, fotografiar o dibujar, una experiencia, una anécdota, palabras, ideas y producciones de los niños y niñas como dibujos, modelados, maquetas, collages, juegos, así como interacciones que los adultos tienen con ellos, o la conformación de espacios por poner algunos ejemplos. En este artículo nos centraremos en la importancia de la observación de los niños y niñas, lo que no quita importancia a lo que es observar los ambientes de aprendizaje, lo que también es relevante permitiendo mejorarlo de acuerdo a los propósitos que se tenga y en definitiva a que los niños y niñas aprendan y se desarrollen en un ambiente que les produzca bienestar y ofrezca una amplia gama de oportunidades de aprendizaje.

Diariamente los educadores tienen buenísimas fuentes de información, las que muchas veces pasan inadvertidas o bien sorprenden, pero no se registran. Por otra parte, muchas veces se confeccionan instrumentos estructurados con gran esfuerzo y los que frecuentemente limitan las observaciones. En cambio, si se observa de manera más "abierta", se registra y se documenta con instrumentos amplios, como registros descriptivos o fotografías, se hace visible los procesos de cada niño y niña. Mediante la observación aprendemos de ellos; aprendemos cuáles son sus intereses y los recorridos que siguen para construir sus conocimientos (Arqueros, 2012).

Es relevante preguntarse ¿qué se está observando? ¿Para qué se observa? ¿Cómo se observa? ¿Quiénes observan? ¿Cuándo se observa?, ¿Qué se hace con la observación? y otras preguntas que nos lleven a la reflexión. A modo de reflexión preliminar abordaremos algunas de ellas para facilitar su discusión.

En cuanto a qué se observa, es importante considerar otros factores del currículo, si se quiere optimizar el proceso de enseñanza y aprendizaje de los niños y niñas, como es la relación del adulto hacia los niños/as, las prácticas pedagógicas, el aula y muchas otras variables que influyen en este proceso.

Para qué se observa, es de relevancia, que no sea una tarea que permita "llenar" instrumentos pre-escritos, ya que se debería observar para mejorar el proceso de enseñanza y aprendizaje, descubrir y aprender de los niños y niñas, sus intereses, sus características, sus habilidades, sus fortalezas.

En relación a cómo se observa, existen criterios al momento de hacerlo, por ejemplo:

- En ambientes naturales, donde no se creen situaciones ficticias. La evaluación es parte del aprendizaje y, por tanto, si el niño y niña aprenden jugando así también debería realizarse la evaluación. Un ambiente natural significa no irrumpir con los que está haciendo el niño o niña, es utilizar y aprovechar sus espacios y tiempos.

- $\quad$ Con otras personas. Es decir, se deben generar espacios para que diferentes agentes educativos que participan en el proceso: educadores, personal técnico, personal de apoyo, familiares y otras personas que están involucradas den sus opiniones, contribuyendo al diálogo, al diagnóstico, a la mejora. En cuanto a la familia es relevante en ello, reconociendo los que sus hijos hacen y van aprendiendo. En el caso de los niños y niñas también pueden observar sus fotografías o evidencias haciendo 
recuerdos de lo vivido, además de demostrarles que lo que han hecho o aprendido, permitiéndoles tener nuevos o consolidar aprendizaje. Cuando van creciendo pueden participar más, haciendo pequeñas apreciaciones o metacognición o transferencia de lo realizado.

- De manera sistemática. Que sea una acción con una clara intención, con un orden y permanencia, donde producto de varias observaciones se pueda analizar, emitir juicio y tomar decisiones.

En tal sentido, la evaluación no puede ser reducida a una simple cuestión metodológica, a una simple "técnica" educativa, ya que su incidencia impacta en el aprendizaje de los niños. Habría por tanto que revisar diversas técnicas e instrumentos que se usan actualmente especialmente en relación a la evaluación formativa donde se registran observaciones, alejándose de aquellas estandarizadas, y privilegiando registros abiertos donde se registre de manera cualitativa.

Respondiendo a la pregunta cuándo observar, se hace necesario hacerlo en todo momento, y no en actividades "especiales" diseñadas como son las experiencias de aprendizaje que se planifican con intencionalidad cada día. Es así como en la muda, la colación, la hora de patio, el lavado de dientes o muchas otras experiencias debiesen ser observadas.

Para Forman y Hall (2005), hay cinco motivos para observar a niños:

- Podemos descubrir sus intereses. Ej. le gusta jugar con la pelota.

- Apodemos evaluar los grados de su desarrollo. Ej. La niña o tira la pelota muy fuerte o no la tira; no varía la fuerza según un continuo de mucha fuerza, menos fuerza y poca fuerza.

- Descubrimos cuáles estrategias utilizan los niños para alcanzar sus metas. Ej. La niña convence a un amigo a que le preste la pelota pasándole otro objeto del interés del niño.

- Nos ayuda a descubrir qué habilidades los niños necesitan practicar y sus logros. Ej. Descubrimos que prefiere jugar a la pelota ya que observamos que al jugar a saltar la cuerda se enredaba.

- Aprendemos de sus personalidades y temperamentos. Ej. cuando le quitaron la pelota se quedó callada y se fue a un rincón de la sala.

Asimismo, y en coherencia con los desafíos de una educación centrada en las personas, donde importa ayudarla en su desarrollo y aprendizajes, se deben privilegiar registros que permitan detenerse en el proceso más que en el producto, especialmente en evaluación formativa. Haciendo alusión a Scriben (Martínez, 2012), quien en la década de los '60 estableció la distinción entre evaluaciones recordemos que "en el discurso se han enfatizado las bondades de la evaluación formativa, aunque en la práctica ésta ha permanecido a la 
sombra de la sumativa", bajo presiones instrumentalistas, y con una mirada hegemónica. (Moreno-Olivos, 2010, p.88)

Por otra parte, existen instrumentos no estructurados o abiertos o llamados también registros de tipo descriptivos, donde no conlleva números, sino que se narra lo observado de manera más objetiva, sin clasificar o categorizar. Hoy en día cuando la educación se centra mucho más en el proceso que en los resultados y donde es necesario reconocer las características, necesidades, intereses y fortalezas individuales, los registros cobran real importancia. Como dice Santos (1998) al respecto, no es aconsejable, la utilización de escalas de observación que desmenucen la realidad para efectuar un registro cuantitativo, porque:

- Cierran la atención de quien observa, no dando cabida a lo impredecible, a lo novedoso, a lo particular, a la singularidad del observado.

- $\quad$ Restringe la realidad al encasillar el comportamiento, sin describir o ampliar el registro de lo observado.

- Descontextualiza la observación, ya que no permite situar cada hecho en el clima y ambiente en que se produce.

- Empobrece, a través del aparente rigor de la cuantificación, la riqueza de matices de la conducta humana tan compleja, limitando lo observado.

Por el contrario, los registros abiertos ofrecen libertad en cuanto a qué observar y permitir registrar hechos incidentales, rescatando la singularidad de los niños y niñas, lo emergente y conllevan aprendizajes de los adultos respecto a los niños y niñas.

Con respecto a la pregunta ¿qué se hace con la observación?, es de relevancia tener presente que sólo después de variadas y reiteradas observaciones se puede llegar a emitir un juicio, igualmente, no es menor qué se hace con lo que se observó y registró, pues muchas veces se guarda en cuadernos o ficheros sin ser el punto de partida para el análisis, interpretación, evaluación y una toma de decisiones, tanto con respecto al planteamiento o selección de aprendizajes esperados, cambios en las estrategias metodológicas, la ambientación u otros que se estimen relevantes.

La documentación pedagógica como señala Alfredo Hoyuelos 2006 es una interesante opción, la que consiste en:

...la recogida y exposición sistemática y estética (a través de escritos, imágenes, paneles, vídeos, palabras de los niños y niñas, productos gráficos) de los procesos educativos. No se trata solo de plasmar lo acontecido, sino de construir -en diversos formatos- un producto público que dé cuenta narrada de lo vivido." Asimismo, agrega Abad (2008) el usar este tipo de estrategia permitirá la reflexión rigurosa y metódica de los procesos educativos. (p.153)

Algunas de las características de la documentación pedagógica, de acuerdo a lo expresado por el italiano Malaguzzi (Hoyuelos, 2006) se caracteriza por: 
- Visibilizar los procesos pedagógicos de los niños y niñas, haciendo público y confrontable el trabajo de la escuela.

- Registrar diferentes tipos de aprendizajes y procesos.

- Auto-representación, entendida como la capacidad de cada espacio individual de narrar el conjunto de elecciones que generan el ambiente de la escuela como un holograma.

- Tener un orden metafórico de los registros documentados.

- Permitir pensar, reflexionar e individualizar los campos de investigación en el proceso de elaboración de cada uno de los proyectos.

Lo importante es hacer de este proceso un medio para comprender a los niños y niñas, no se documenta para archivar o guardar en álbumes, se documenta para llevar registro, para hacer un trabajo sistemático y riguroso. Como decía Malaguzzi, "lo que no se ve no existe" (en Abad, 2008) estimulando a algunas educadoras a observar y registrar. Se ofrece como una ocasión para releer los procesos de aprendizaje (Abad, 2008, p.64) y no solo a los educadores, sino que a los niños y niñas les permite recordar lo vivido, tomar conciencia de los procesos vividos, a revivir la situación. En cuanto a las familias, les permite conocer lo que hacen sus niños y niñas dentro del centro educativo, hablar de ello, recrearlo en el hogar y valorar el trabajo pedagógico que desarrollan los educadores.

De esta manera se puede utilizar material audiovisual, el que permite recolectar evidencias como fotos o dibujos y observarlas, constituyendo un potente material para hacerse preguntas, analizar, posteriormente tomar decisiones después de diversas experiencias observadas garantizando la confiabilidad de lo recogido y posteriormente las decisiones.

\section{PALABRAS FINALES}

Finalmente, la evaluación es un problema ético ya que como dice Miguel Ángel Santos Guerra, "dime como evalúas y te diré qué tipo de profesional y persona eres" (2014) señalando que más que un proceso de naturaleza técnica es una actividad que contiene dimensiones psicológicas, políticas y éticas. Nos preguntamos ¿evaluar éticamente es posible? Ello no siempre depende de la persona que está desarrollando el proceso evaluativo, "muchas de nuestras acciones profesionales se enmarcan en instituciones y son colaborativas de ellas, es decir, nosotros no somos los únicos responsables de la acción." (Fontecilla, 2015, p. 223), sin embargo, debemos con identidad y convicción profesional demostrar las implicancias que tienen para las personas los actos pedagógicos y entre ellos los evaluativos, aportando al desarrollo humano, ya que como señaló Hopkins (1989), la evaluación es buena sólo si sirve para enriquecer plenamente a las personas que en ella intervienen. 
Esperando que este artículo no lo encuentre el lector, ni excelente, ni bueno, ni logrado, y sólo sea un medio para una posterior discusión de este trascendente tema, contribuyendo a una posible mejora en el quehacer de una educadora o educador que está en permanente reflexión, discusión y construcción en pos del bienestar, aprendizaje y desarrollo de los niños y niñas.

\section{REFERENCIAS BIBLIOGRÁFICAS}

Abad, J. (2008). Iniciativas de educación artística a través del arte contemporáneo para la escuela infantil (3-6 años). (Tesis doctoral). Universidad complutense de Madrid. ISBN: 978-84-692-4259-9

Arqueros, J., Balaguer, I. y otros. (2012) Documentar, una mirada nueva. Temas de infancia $\mathrm{n}^{\circ}$ 29. Educar de 0 a 6 . Rosa Sensat. Barcelona: Octaedro.

Davoli, M. (2011) Documentar la vida de los niños y niñas en la escuela. Temas de infancia. $\mathrm{N}^{\circ} 28$ educar de 0 a 6. Rosa Sensat. Barcelona: Octaedro.

De Ketele, J. M., (1984). Observar para educar, Madrid: Aprendizaje-Visor.

Fontecilla, P. (2015) Ética para educadores. ¿Cuál es el lugar de la ética en el siglo XXI? (1ª․ Ed) Santiago de chile: Editorial universitaria.

Forman, G. y Hall, E. (2005) preguntas compartidas. La importancia de la observación en la educación de niños pequeños. Vol. $7 \mathrm{~N}^{\circ} 2$. Videatives, inc. Amherst (Massachusetts) en http://ecrp.uiuc.edu/v7n2/forman-sp.html.

Himmel, E. y otros. (1999). Hacia una evaluación educativa. Aprender para evaluar y evaluar para aprender. Volumen I conceptos actuales sobre la evaluación del aprendizaje escolar. Santiago de Chile: Pontificia Universidad Católica de Chile.

Hopkins, D. (1989). Evaluation for school development. Londres: Open University Press.

Hoyuelos, A. (2007). Documentación como narración y argumentación. Pamplon: aula de infantil $\quad \mathrm{N}^{\circ} \quad 39 . \quad$ Disponible en http://caps.educacion.navarra.es/infantil/attachments/article/112/documentacion -como-narracion-y-argumentacion.pdf

Manhey, M. (2009). Evaluación en educación infantil. Universidad Central. Santiago: LOM

Manhey, M. (2014). Currículo en educación infantil y diversidad: un tema pendiente. Disponible en: $w w w . r e d a l y c . o r g / a r t i c u l o .0 a ? i d=65848281006$. ISSN: $1316-0087$

Martínez Rizo, F. (2012). La evaluación formativa del aprendizaje en el aula en la bibliografía en inglés y francés. Revisión de literatura. Revista Mexicana de Investigación Educativa, 17 (54), 849-875. 
Moreno-Olivos, T. (2010). Lo bueno, lo malo y lo feo: Las muchas caras de la evaluación. México: en Revista Iberoamericana de Educación Superior (RIES), vol. I, núm.2, pp. 8497, http://ries. Universia.net/index.php/ries/article/view/55/evaluacion [consulta: 20 agosto 2018).

Perrenoud, P. (2010). La evaluación de los alumnos. De la producción de la excelencia a la regulación de los aprendizajes entre dos lógicas. Buenos Aires: Colihue.

Santos, M. (1998). Evaluar es comprender. Argentina. Editorial Magisterio del Río de la Plata.

Santos, M. (2014). La evaluación como aprendizaje, cuando la flecha impacta en la diana. Madrid: Narcea.

Turri, C. (2004). Evaluar desde el comienzo. Ediciones novedades educativas. México. 